\title{
Business model et création d'entreprise
}

La notion de business model est apparue en contexte entrepreneurial avec l'avènement de ce qui a été qualifié de start-up. L'article en propose une conceptualisation pédagogiquement déclinée dans le cadre de programmes de sensibilisation et de formation à l'entrepreneuriat. Le cadre théorique a également été transféré pratiquement pour servir l'accompagnement des porteurs de projets, montrant ainsi que le concept de business model est pertinent pour aider les créateurs à comprendre le cœur de leur affaire. Le plan d'affaires, apparaît alors comme une version rédigée du projet, mais la conviction repose avant tout sur le business model expliquant comme la valeur est générée, rémunérée et partagée. Autrement dit, le business model est une convention d'affaires et le plan d'affaires un document rédigé pour la communiquer. 
I 1 n'est pas étonnant de parler de Business model (BM dans la suite du texte) en contexte de création d'entreprise puisque c'est pour aider à comprendre les projets d'affaires envisagés sur internet, média alors nouvellement apparu, que les parties prenantes potentielles au projet, au premier rang desquelles les investisseurs, ont exigé un effort de conceptualisation supplémentaire de la part des créateurs d'entreprise. Si, aujourd'hui, on sait comment quelques entreprises remarquables ont construit leur modèle d'affaires (ebay, Amazon, etc.), il était moins aisé, lors de la prise de conscience du potentiel offert par internet, d'imaginer comment y réussir en affaires. Ce problème du succès en contexte de forte incertitude a très largement dépassé les frontières des nouvelles technologies de l'information et de la communication pour, dans un premier temps, concerner les différentes formes d'innovation, puis tous les projets entrepreneuriaux, que ces derniers soient innovants ou pas. Il est même désormais fréquent de discuter du BM avec le porteur d'un projet même lorsque la dynamique qu'il impulse n'est pas réservée à la création d'une firme. Les projets peuvent en effet concerner différentes sphères professionnelles (art, culture, économie sociale et solidaire, sport, etc.) au sein desquelles l'observation montre des individus entreprenant selon différentes voies (exemple : reprise d'entreprise, création d'une activité au sein d'une forme organisationnelle existante, etc.), pour différentes sphères (privée, publique, etc.) et donnant lieu à l'émergence de différentes formes (sociétaire, associative, réticulaire, etc.).
L'utilisation de l'expression se diffuse, avec une croyance en une acception partagée ${ }^{1}$. Or, la participation à divers jurys de sélection de projets permet de remarquer que les protagonistes (investisseurs privés, conseillers, prêteurs institutionnels, etc.) interrogent le BM sans se soucier de savoir si chacun des membres présents autour de la table lors de ces comités possède la même acception du concept et, conséquemment, de l'affaire envisagée. En première écoute, les praticiens ont tendance à réduire le BM aux sources de revenus. Mais, dépassant ce cadre restrictif, leurs interrogations portent également sur l'apport du projet à l'ensemble des acteurs susceptibles d'être concernés voire impliqués (c'est-à-dire les parties prenantes ou stakeholders). Autrement dit, le BM est une notion dépassant la seule explication des sources de revenus au sens strict du terme pour recouvrir l'ensemble des revenus partagés, ceux-ci ne se traduisant pas forcément par une valeur pécuniaire (par exemple, un investisseur institutionnel peut attendre que le projet soutenu crée des emplois).

Quoi qu'il en soit, entre autres lors de la tenue de ces jurys et parfois avant qu'un business plan (ou plan d'affaires) ne soit finalisé, le créateur doit convaincre les parties présentes d'adhérer à sa proposition de lancement d'une affaire. La plupart du temps, il s'agit de lever des fonds permettant de démarrer ou d'aller plus loin dans la mise au point du projet de création (par exemple, dans ce dernier cas, obtenir une enveloppe financière permettant d'engager le développement d'un prototype, de commander une recherche en propriété indus-

1. Le BM est parfois traduit par modèle d'affaires ou par modèle économique. Cette dernière terminologie n'est pas toujours considérée comme reflétant correctement la notion. 
trielle ou une étude de marché plus étayée, etc.). Mais plus largement, les ressources à réunir sont de diverses natures (tangibles ou intangibles, cognitives ou matérielles, financières ou non financières, etc.) et tout porteur d'un projet de création d'entreprise déploie un exercice de conviction visant à recevoir l'adhésion des possesseurs de ces ressources pour en faire des parties prenantes. Pour démarrer, il faut qu'un ensemble d'acteurs s'accorde à collectivement reconnaître que le modèle d'affaires proposé est pertinent. Le mimétisme intervient en partie car cette reconnaissance est influencée par la croyance en l'adhésion des autres. Il y aurait une forme de représentation collective autour de ce qu'est, ou doit être, ou peut être le BM. Celui-ci serait alors une convention en construction qu'il faudrait, en quelque sorte, implanter au sein d'un système d'offre. Tout porteur d'un projet aurait intérêt à connaître la notion afin de mieux préparer son exercice de conviction.

De façon pratique, le conseiller en création d'entreprise peut alors s'interroger sur l'utilisation du BM comme outil de mise au point du projet. Aujourd'hui, c'est la notion de plan d'affaires qui guide sa méthodologie de création d'entreprise. Sans rejeter ce que ce document écrit apporte à la compréhension du projet, celle de BM peut en amont s'avérer utile dans la construction de celui-ci. Le chercheur intéressé par le conseil en création d'entreprise peut formuler la question suivante: " la réflexion en termes de BM est-elle en mesure d'aider le créateur d'entreprise dans le cadre de la mise au point de son projet et des exercices de conviction qu'il est amené à déployer? ». La question n'est pas triviale, car jusqu'à présent la plupart des créateurs d'entreprise se sont passés de la notion de BM, et plus encore d'une conceptualisation de celle-ci. Mais de nombreux créateurs ne bénéficient pas du conseil des structures spécialisées, alors qu'il est démontré que les entrepreneurs accompagnés ont très sensiblement plus de chance de réussite que les autres (Birley et al., 1992). Cet article s'intéresse à la mise au point d'une méthode concrète d'élaboration d'un BM afin d'apprécier sa pertinence, aussi, dans une pratique de conseil et d'accompagnement.

La première revue de littérature effectuée pour cerner l'objet de nos recherches fait apparaître que d'une discipline à l'autre (ex: entrepreneuriat, finance, système d'information, stratégie, etc.), les conceptions académiques du BM diffèrent, même s'il reste possible de relever quelques lignes communes. Nous avons abouti à une conception où le $\mathrm{BM}$ est considéré comme la première convention autour de laquelle des possesseurs de ressources se rejoignent et prennent partie au projet. Le BM est la représentation d'une affaire exprimant comment la valeur est générée, rémunérée et partagée avec, pour ces trois dimensions, des questions relatives aux volumes (de génération, de rémunération, de partage) et aux canaux (par quelles voies la valeur estelle générée, rémunérée, partagée?) C'est en cela que cette représentation doit devenir une convention, c'est-à-dire une conception collective forcément influencée par les attentes des parties prenantes (notamment les porteurs du projet, les clients, les financeurs, etc.) La théorie des conventions est ainsi centrale dans notre conceptualisation et le présent article s'y focalise même si deux autres corpus théoriques guident également notre démarche: celui des ressources et celui des parties prenantes. Ils 
aident, d'une part, à cerner la constitution $\mathrm{du}$ BM (quelles ressources réunir pour le projet? Qui possède celles-ci? Quels possesseurs de ces ressources faut-ils lier au $\mathrm{BM}$ ?, etc.) et, d'autre part, à comprendre comment le BM procure un avantage concurrentiel (quel agencement des ressources réunies pour construire un avantage concurrentiel? Comment lier durablement les parties prenantes au projet?, etc.). $\mathrm{Ce}$ dernier point établit un pont entre le domaine de l'entrepreneuriat et celui de la stratégie, finalement liés autant dans le domaine théorique que dans la pratique.

Dans la première section, nous expliquons que la théorie des conventions permet de comprendre comment des possesseurs de ressources s'accordent autour d'une convention donnant naissance à l'organisation. Notre conceptualisation s'est peu à peu affirmée par les allers-retours entre la théorie et la pratique, au travers de différents protocoles relevant de la rechercheaction. La deuxième section et la troisième section présentent, de façon non exhaustive, nos terrains, respectivement pédagogique et pratique, en contexte de création d'entreprise auquel se circonscrit notre travail.

\section{Conceptualisation du BM en contexte de création d'entreprise}

Le BM est ici une convention construite autour d'une affaire envisagée (et plus tard effective) à laquelle prend plus ou moins directement part un ensemble de partenaires (les parties prenantes) apportant des ressources en échange de ce qu'ils attendent de la relation instaurée.

S'agissant des ressources, le créateur ne peut démarrer son affaire sans, à la fois, identifier puis réunir ce dont il a besoin et sans montrer ce qu'il va en faire (la façon dont il va les agencer, donc les organiser, pour en tirer des compétences). Handicapé par l'inexistence d'un passé dont les possesseurs de ressources tirent plus habituellement des renseignements (en termes de solvabilité, de qualité, etc.), la firme non encore née s'appuie sur un ou des porteurs devant, outre leur propre expérience, montrer comment les apprentissages des premières années d'exploitation vont conduire à une maîtrise de l'organisation des ressources réunies. La configuration organisationnelle mise en place joue alors un rôle crucial, puisqu'elle agence ces ressources de façon optimale, c'est-à-dire de sorte à ce que la valeur de l'offre soit appréciée, d'une part, par les marchés (valeur générique du projet) et, d'autre part, par les différentes parties prenantes (valeur qui leur est singulièrement apportée).

S'agissant de ces parties prenantes, la dynamique inhérente à l'impulsion d'une organisation suppose une énergie déployée auprès de possesseurs de ressources (en premier lieu l'entrepreneur lui-même) qu'il faudra durablement satisfaire pour les maintenir en relation avec l'ensemble construit par la convention d'affaires proposée. Le BM est une cristallisation des relations entre parties prenantes. En effet, pour que les affaires puissent démarrer, il faut apporter une valeur attendue par les parties prenantes en compensation de ce qu'elles consentent (et qui leur confère un pouvoir plus ou moins grand, ce qui peut poser des problèmes de gouvernance). Pour cela, le créateur doit montrer aux possesseurs de ressources qu'il souhaite « embarquer » dans ses affaires ce qui constitue le cœur de son business, c'est-àdire le modèle avec lequel il compte apporter de la valeur qu'il saura se faire rémuné- 
rer par le marché. Ce modèle doit être suffisamment clair pour que chacun s'accorde à reconnaître que, effectivement, c'est là une bonne façon de générer de la valeur qu'un chiffre d'affaires, payé, récompensera. Les mêmes parties prenantes sauront mieux, à l'issue de cette démonstration, expliciter ce qu'est le véritable business de l'entreprise (l'acteur en possédant la représentation la plus complète reste le créateur). En échange des ressources apportées au créateur, leurs possesseurs, alors devenus parties prenantes, attendent eux-mêmes une rémunération, c'est-à-dire quelque chose en échange de ce qu'ils apportent (ressources contre ressources). Le BM doit montrer de façon concrète comment l'argent va rentrer et, de façon plus abstraite, comment vont se dérouler les relations d'échange avec les parties prenantes. Mais pour qu'un possesseur de ressources se transforme en stakeholder d'un projet de création d'entreprise, que le processus en soit au début ou déjà bien avancé, il lui faut adhérer à la proposition du créateur et croire en sa pérennité. Cette proposition peut être comprise par la théorie des conventions. Il est utile de rappeler quelques fondements de cette théorie avant de présenter les questions posées par sa mise en œuvre dans le cadre qui nous intéresse ici.

La convention constitue un moyen d'ajustement des comportements intersubjectifs (Gomez, 1994), un moyen de coordination des agents basé sur des dispositifs cognitifs collectifs (Munier et al., 1993). Elle est une procédure régulière de résolution de problèmes collectivement établie faisant appel à une rationalité procédurale; néanmoins, elle se présente aux acteurs sous forme objectivée (Dupuy et al., 1989). Elle doit « être appréhendée à la fois comme le résul- tat d'actions individuelles et comme un cadre contraignant les sujets " (ibid., p.143). Elle permet de comprendre comment se constitue une logique collective et comment les comportements des membres d'une population peuvent faire preuve d'une certaine régularité dans une situation récurrente (Orléan, 1994). Elle suppose des conditions fixées de conformité et de croyances régies par une base de connaissances communes quant aux comportements des autres.

La théorie des conventions explique ainsi que le comportement des individus est lié à un univers symbolique établissant les règles $\mathrm{du}$ jeu économique. Cet univers est constitué de représentations partagées permettant d'ériger des normes de conduites économiques et sociales. Ainsi, globalement, le comportement d'un individu résulte de ce qu'il croit être le comportement des autres dans une situation et à un moment donnés. L'individu se réfère à ce qu'il connaît des décisions prises par les autres ou à ce qu'il imagine être leur décision, persuadé qu'il est de savoir comment ils vont réagir face à une situation d'incertitude: « une convention apparaît dans une situation d'incertitude radicale (...) elle est une régularité qui résout de manière identique des problèmes de coordination identiques. Elle se substitue alors au calcul individuel. »(Gomez, 1994, p. 95). À ce titre, le BM compose forcément avec les conventions déjà à l'œuvre dans l'environnement, que le créateur les respecte ou qu'il propose une nouvelle vision des affaires qui devra devenir une convention, par exemple une innovation. Ce dernier cas est délicat au regard du rôle du mimétisme dans le comportement des agents: "la convention est une construction sociale, 
parce qu'elle n'existe concrètement que par l'accumulation de comportements mimétiques, auxquels elle donne, comme un miroir social, leur sens. " (Gomez, 1996, p. 145). Le BM est assimilable à une convention première à laquelle l'entrepreneur doit faire adhérer des partenaires (organismes, institutions ou individus) possédant les diverses ressources nécessaires ou utiles au projet. En raison du rôle du mimétisme, ces derniers sont d'autant plus facilement convaincus que d'autres, avant eux, ont déjà adhéré au projet. Par exemple, démontrer que des clients sont convaincus par la valeur de l'offre et sont prêts à s'engager dans l'achat, provoque un premier effet d'entrainement susceptible de recevoir l'adhésion d'autres catégories de parties prenantes potentielles (capitalrisque, banque, etc.). Ainsi, on peut imaginer un processus progressif d'adhésion au registre conventionnel proposé par l'entrepreneur (ou l'équipe entrepreneuriale). Ce que les partenaires doivent saisir, c'est le cœur de l'affaire, ce qui fait accord ou qu'ils croient faire accord. Le BM devient en quelque sorte l'unité élémentaire d'un véritable business naissant (Jouison, 2005). Il évolue en fonction des interactions de l'entrepreneur avec des possesseurs de ressources puis, plus tard, de l'entreprise avec ses parties prenantes. Ainsi, toute organisation naissante développe progressivement un registre conventionnel qu'elle va partager avec l'ensemble de ses parties prenantes (Verstraete, 1999, 2003). Le BM est une convention évolutive. Les conventionnalistes précisent que « Les conventions sont des construits stables mais pas statiques (...) les conventions évoluent, se modifient et parfois disparaissent. " (Gomez et al., 2000, p.701). La partie stable des conventions confronte, en contexte de création d'entreprise, l'entrepreneur à des conventions de niveaux distincts bien que liés :

- Le premier, relatif à tout projet d'entreprendre, est de montrer l'intensité et la qualité du travail fourni dans le cadre du processus entrepreneurial en le formalisant dans un business plan. « Le plan d'affaires (ou business plan) est la forme écrite de l'exercice de conviction communicant la vision stratégique du porteur de projet (ou des porteurs de projet) et montrant que le modèle envisagé peut générer suffisamment de valeur partageable pour être soutenu par la partie à laquelle le document est adressé, et dont des ressources sont attendues. Il inscrit le projet dans le temps par l'explicitation des ressources nécessaires et employées pour atteindre les objectifs et, ainsi, réaliser la vision. » (Verstraete et al., 2006, p. 380). Dans le «monde » de la création d'entreprise, un certain nombre d'acteurs essentiels au démarrage de l'affaire, notamment les investisseurs et les prêteurs de capitaux, exige ce document qui oblige, également, le créateur à formaliser son projet, par exemple en expliquant le montage juridique, les tableaux financiers prévisionnels, etc. Il ne peut donc être assimilé au $\mathrm{BM}$, bien qu'il doive être construit autour de ce dernier.

- Le deuxième est relatif au secteur d'activité au sein duquel les entreprises ont leur conception de ce que sont les affaires et, donc, des conventions correspondantes. Sans forcément s'inscrire dans la théorie des conventions, la littérature a, assez largement, mis à jour les représentations, les conceptions ou les valeurs guidant les dirigeants de certains secteurs (pour une synthèse, voir Desreumaux, 1995). 
- Le troisième registre est relatif aux détenteurs de ressources sollicités, lesquels partagent des conventions relatives à leurs métiers et que l'entrepreneur doit connaître afin d'être entendu d'eux. Par exemple, il est utile de connaître ce qui guide un capital-risqueur dans le suivi d'un dossier pour mieux savoir négocier avec lui. Le BM est une convention s'encastrant dans d'autres registres conventionnels dont le porteur du projet doit tenir compte.

Autrement dit, le créateur doit « faire naître », ou concevoir une convention, certes potentielle au départ, autour de laquelle des possesseurs de ressources vont s'accorder pour y voir une bonne façon de faire des affaires en pariant que le projet régulera de façon optimale les échanges de valeur (autrement dit de ressources) escomptés entre toutes les catégories de parties prenantes. Un réseau se crée ainsi autour du BM proposé.

La figure 1 récapitule notre conceptualisation en suggérant ce que cela implique pratiquement dans la construction d'un BM convaincant devenant ainsi une convention. Sa partie haute comporte les éléments

Figure 1 - Le BM comme convention d'affaires

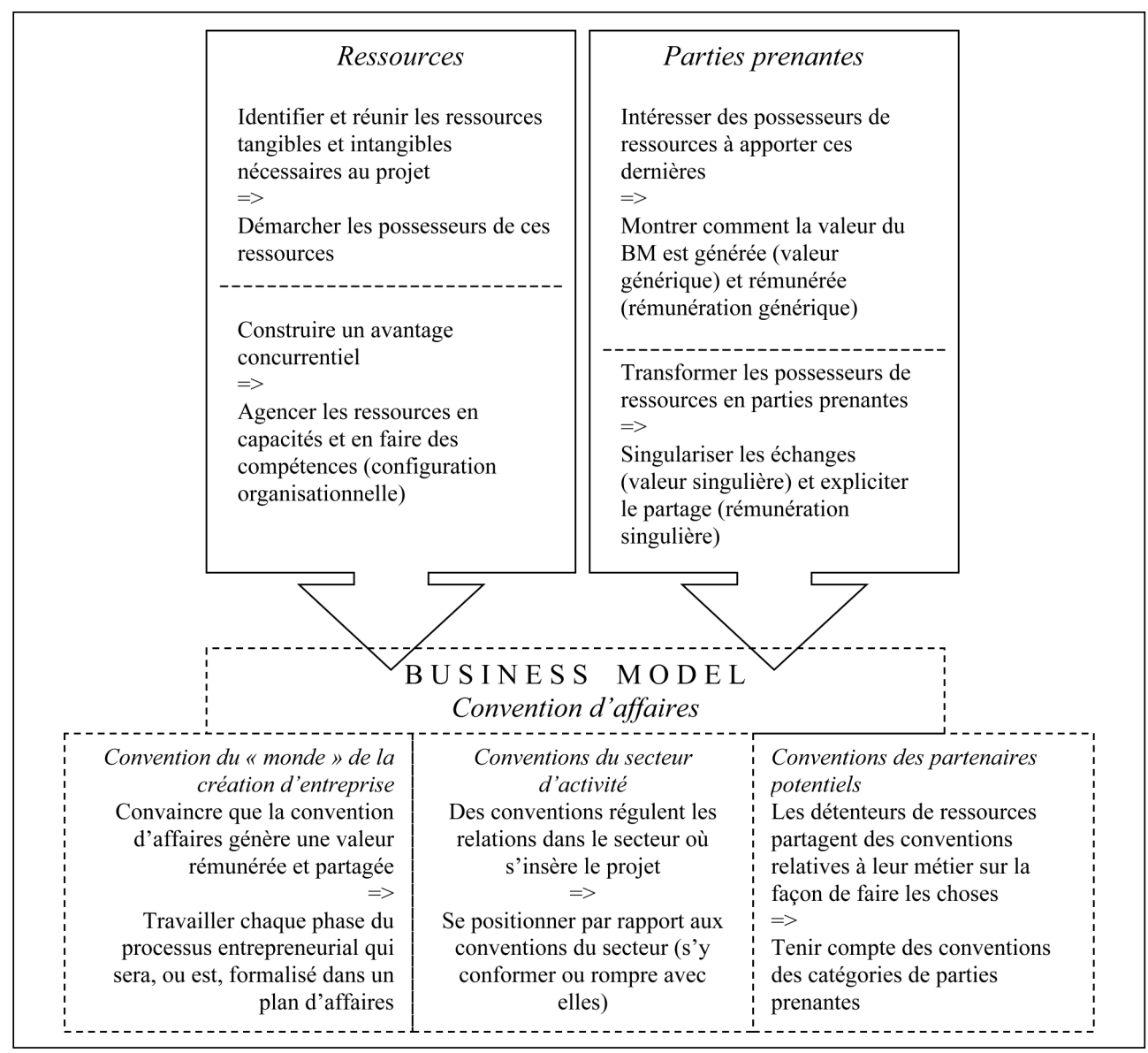


constitutifs du BM (qui s'exprimeraient dans un processus forcément itératif) et sa partie basse montre que la convention d'affaires doit composer avec d'autres registres conventionnels, chacun d'entre eux étant poreux ou encastré dans les autres.

Le BM peut être rapproché de ce que les conventionnalistes nomment une « convention d'effort ». La théorie des conventions définit l'entreprise comme une organisation permettant de normaliser les relations entre les parties prenantes intéressées par sa survie dans la logique de contribution à un objectif commun (Gomez, 1997). Il s'agit d'un lieu de confrontation collective à l'incertitude dans une perspective de rentabilité (Ughetto, 2000). Cette dimension collective de l'organisation implique l'existence d'une convention d'effort partagée par les parties prenantes sur laquelle elles fondent leur action, l'effort commun ainsi fourni permettant de donner « corps » à l'entreprise. Dans cette veine, une entreprise (convention d'effort) peut reposer sur une seule convention d'affaires mais également sur plusieurs. Par ailleurs, le Business Model n'est pas strictement assimilable à la convention d'effort car il intègre en partie la notion de « convention de qualification ». Les conventions de qualification correspondent au marché (Gomez, 1997). Les échanges s'y déroulant sont définis au regard de conventions portant sur la qualité des acteurs engagés dans cet espace d'échange c'est-à-dire sur les comportements normaux, connus et partagés, auxquels chacun fait référence pour agir, attendant des autres acteurs du marché qu'ils fassent de même.

Sur cette base théorique, nous avons construit une méthode servant la pédagogie et la pratique de la conception d'un BM.

\section{Insertion du BM dans une pédagogie de l'entrepreneuriat}

Qu'il s'agisse d'une sensibilisation à l'esprit d'entreprendre ou d'une formation dédiée à l'entrepreneuriat, les étudiants sont souvent placés dans des situations concrètes de défense d'un projet de création d'entreprise devant un jury composé de professionnels (banquier, capital-risqueur, investisseur institutionnel, conseiller en création, etc.). Il est demandé à ces derniers de reproduire en salle de classe ce qu'ils vivent dans leur pratique d'évaluation de projets de création. En 2002, pour la première fois, certains étudiants furent explicitement interrogés sur leur BM. La notion était certes évoquée en cours, mais n'avait pas encore fait l'objet d'un enseignement spécifique. Il convenait de décliner dans la pédagogie les éléments permettant aux étudiants de cerner le concept et de s'en servir pratiquement dans la mise au point de leur projet. La question générique à laquelle nous nous intéressons ici ( la réflexion en termes de BM est-elle en mesure d'aider le créateur d'entreprise dans le cadre de la mise au point de son projet et des exercices de conviction qu'il est amené à déployer? ») trouvait ainsi un terrain propice à l'expérimentation. Les manuels traitant du BM sont à la fois peu nombreux et muets en matière d'apport pédagogique. La question devenait dans ce contexte: comment aider les étudiants à comprendre le $\mathrm{BM}$, puis à construire ce registre conventionnel aidant à convaincre?

Notre expérimentation a porté, dès l'année 2003, principalement sur le terrain de trois programmes bordelais: un troisième cycle universitaire consacré à l'entrepreneuriat, un deuxième cycle 
universitaire, une première année de trois programmes en École de commerce $^{2}$. D'autres programmes bénéficient désormais de l'ingénierie mise en place, en France et à l'étranger, dispensés par divers membres de l'équipe d'enseignants chercheurs. Aujourd'hui, environ 3000 étudiants ont été formés au BM selon la conception ici présentée.

Préalablement à l'insertion du BM dans le programme pédagogique, nous avons procédé à quelques entrevues avec des conseillers expérimentés en création d'entreprise (dont le créateur de plusieurs structures d'accompagnement), notamment pour savoir si la notion de BM était utilisée par eux (entre autres pour apprécier la possibilité de décliner la pratique dans la pédagogie). Force fut de constater que le BM n'était pas explicitement mobilisé, ni même autant théoriquement que concrètement assimilé; le référent instrumental de leur activité restant le business plan. Le problème de la conception d'une pédagogie du BM était posée, sans pour autant remettre en cause ce qui fonctionnait bien, mais en y incluant les conceptions modernes d'évaluation de projet de création d'entreprise ${ }^{3}$. Autrement dit, il convenait d'insérer le BM dans une pédagogie plus complète de l'entrepreneuriat dont il ne peut raisonnablement pas se déconnecter.
Une telle pédagogie peut s'appuyer sur deux lectures complémentaires.

La première consiste à comprendre le phénomène entrepreneurial, notamment les enjeux et les problèmes qu'il pose ainsi que sa genèse et ses niveaux d'analyse. Selon cette perspective, le BM constitue le premier élément de l'organisation naissante puisque la convention conduit à agencer ou à imaginer l'agencement des ressources dans une configuration organisationnelle imbriquée à une organisation plus vaste l'entourant et constituée, à la fois, d'autres conventions avec lesquelles elle compose et d'acteurs dont certains sont les parties prenantes apportant les ressources nécessaires au projet. Cette lecture est essentiellement théorique même si des exemples pratiques jalonnent l'exposé fait en classe. Elle est utilisée dans le programme de troisième cycle.

La seconde lecture, effectuée dans tous les programmes, est plus pratique pour aider les étudiants à comprendre cette convention d'affaires. Elle est processuelle et itérative plutôt que linéaire même si l'axe de temps y prend utilement place. Elle comporte cinq phases génériques: idée, opportunité, $\mathrm{BM}$, vision stratégique et plan d'affaires (Verstraete et al., 2006). Nous nous y attardons ici car elle prépare les déclinaisons pédagogiques et pratiques du BM. Il

2. Les cycles universitaires sont le Master II CREE (création, reprise d'entreprise et entrepreneuriat), d'un volume de 360 heures et l'option entrepreneuriat (70 heures) du Master I « Management» du pôle universitaire de sciences de Gestion de l'université Montesquieu Bordeaux IV. Les trois programmes de Bordeaux École de management sont ESC, SUPT'G et EBP (15h par séminaire). Le PRES université de Bordeaux lance une Maison de l'entrepreneuriat s'appuyant sur la pédagogie ici présentée.

3. Le plus surprenant est que, à l'époque, les porteurs de projet désirant convaincre des partenaires (essentiellement lors de la levée de fonds) se voyaient questionnés sur un concept auquel ils n'étaient absolument pas formés, ni parfois même leurs conseillers. Cette remarque reste en partie d'actualité et une recherche serait particulièrement intéressante pour comprendre comment la notion se diffuse et s'apprend par les confrontations et les pratiques. 
ne s'agit pas de dire que le processus entrepreneurial est balisé, pour son démarrage, par l'idée et, pour sa fin, par le plan d'affaires. Par contre, chacune des étapes identifiées nécessite un travail pouvant bénéficier d'une formation et d'un accompagnement dans le montage d'un projet de création d'entreprise. Dans une certaine mesure, le processus évoque un dispositif gigogne où chaque étape compose la suivante (idée dans opportunité, opportunité dans BM, etc.).

Ainsi vue, la compréhension du BM est d'abord servie par une présentation du processus complet (de l'idée au plan d'affaires). Outre l'étude du couple créateur -projet, le processus comportant cinq phases, aux frontières poreuses, est ici juste résumé dans le but de montrer la place centrale qu'y occupe le BM.

Le porteur du projet doit trouver une idée d'affaires qu'il met au point et, si possible, qu'il protège (exemple : brevet). Il évalue la capacité de cette idée à rencontrer un marché et, ainsi, devenir une opportunité. Il élabore son BM, puis sa vision stratégique qu'il formalise dans un plan d'affaires. Le BM prend ainsi place au milieu du processus, car il est difficile de le concevoir sans avoir approché le marché, lequel ne se limite pas au potentiel de clients mais inclut tout le système d'offre. Il est très lié à la vision stratégique dont il est la principale composante mais il évacue certains aspects que la stratégie ne peut éluder (exemple : le contrôle de la performance). Le plan d'affaires est la version rédigée de cette vision. Il n'est pas qu'un ensemble de mots couchés sur le papier car il consacre toute l'activité menée en amont de sa défense, c'està-dire tout le travail effectué sur chacune des phases, et présente pourquoi et com- ment, concrètement, il est probable que les événements se déroulent comme les prévisions, en partie chiffrées, l'estiment. Mais pour comprendre ces prévisions, il faut préalablement saisir le cœur de l'affaire, le BM. En amont de celui-ci, le travail sur l'idée et sur l'opportunité fait l'objet d'une méthodologie éprouvée, même si parfois, dans le cadre d'une activité nouvelle, l'estimation du chiffre d'affaires reste spéculative. Quant au BM lui-même, la pédagogie implantée s'est largement inspirée de la figure 2.

L'idée et l'opportunité sont, dans une perspective processuelle, les composantes minimales du BM. Mais pour que les étudiants comprennent la convention, où la dimension collective est essentielle, il convient de schématiser les relations avec les possesseurs de ressources. L'une des difficultés majeures d'apprentissage du BM consiste à montrer au porteur du projet qu'il n'est finalement pas seul dans l'élaboration d'un $\mathrm{BM}$ et qu'il convient de rencontrer les possesseurs de ressources avant même que le plan d'affaires ne soit formalisé.

La partie supérieure de la figure 2 explique que le BM ne peut s'imaginer sans l'idée et ne peut convaincre sans montrer qu'une opportunité d'affaires a été en partie évaluée. Plus encore, le porteur du projet doit exposer sa capacité à construire, par l'organisation naissant du phénomène, les compétences permettant de prendre un avantage compétitif. Mais ces compétences ne peuvent être réalisées sans avoir réuni les ressources tangibles et intangibles possédées par des acteurs dont le créateur aimerait qu'elles prennent part aux affaires (voir la partie inférieure du schéma). Si ces possesseurs de ressources perçoivent la valeur générique apportée par le modèle 
Figure 2 - Lecture pédagogique du BM

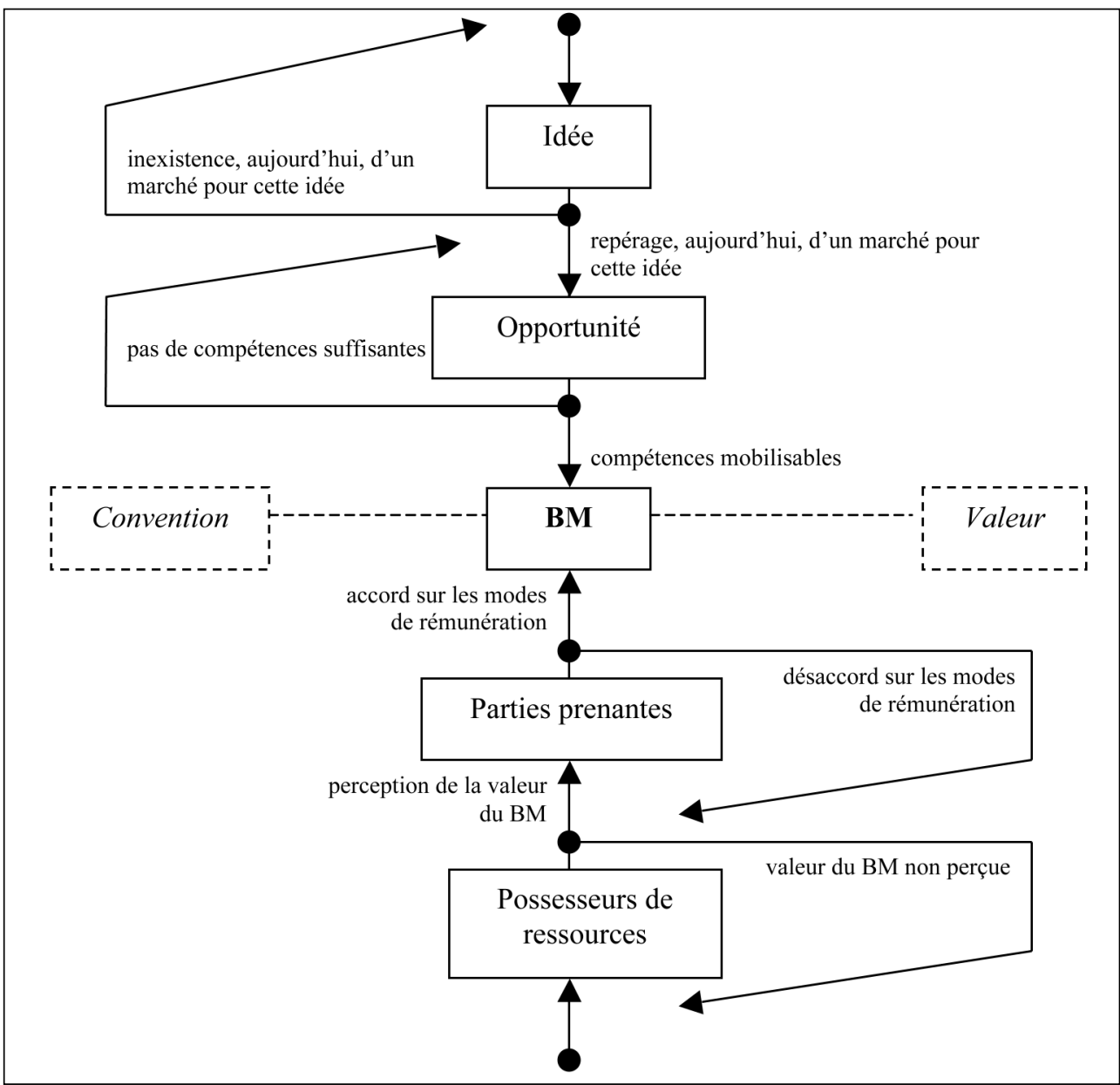

Source : Verstraete et Saporta, 2006.

imaginé (essentiellement la capacité à générer un chiffre d'affaires, donc à savoir exploiter l'opportunité appréciée), ils sont alors susceptibles de devenir des parties prenantes du projet. Mais ils ne le deviendront véritablement que si la valeur d'échange qui leur est singulièrement proposée leur convient (le « ressources contre ressources » évoqué dans la section précédente). Plus exactement, ils doivent comprendre comment la valeur générique est, à la fois, rémunérée (volume et canal de la rémunération) puis répartie entre les différentes parties prenantes (valeur singulière à chacune d'entre elles), sachant que chacune de ces dernières reste avant tout sensible à ses propres attentes. À ce titre, les possesseurs de ressources influencent le BM, tout porteur de projet devant tenir compte, dans sa modélisation, des attentes des différentes parties prenantes. 
Avec ce schéma, l'étudiant comprend que le $\mathrm{BM}$ est une construction collective, en fait une convention devant faire accord et sur laquelle se fondera la future convention d'effort (l'entreprise). Le BM s'articule finalement autour de la question suivante: comment la valeur est-elle générée, rémunérée et partagée? Étant entendu que la valeur revêt deux dimensions, la valeur pour le marché et la valeur pour les parties prenantes.

Si le BM est une convention, ou une forme de représentation collective, et si les propos précédents, adaptés au public et agrémentés d'exemples, permettent une première approche, reste posée la question de l'instrumentation de sa construction. La formalisation est une aide difficilement contournable pour un public ayant besoin de « voir » le résultat de l'avancée de son travail, son « modelage »... À cette fin, et en prenant garde qu'il n'y ait pas d'amalgame entre BM et business plan, en nous appuyant sur les développements schématisés par les figures 1 et 2 , les étudiants sont conduits à remplir un tableau, présenté dans la prochaine section, nous servant également dans l'accompagnement des porteurs de projet.

Dans nos formations, tous les étudiants sont placés en situation d'entreprendre, qu'ils soient ou non porteurs d'un projet et défendent, en fin de programme, leur travail devant un jury. Même en élaborant un projet fictif, l'apprentissage est alors très utile à l'étudiant désirant travailler dans le conseil ou dans l'évaluation de projet (exemple: en banque, en capital risque, etc.). Mais c'est principalement vis-à-vis des créations en cours que l'instrumentation revêt une importance capitale puisque, éthiquement, les enjeux ne sont pas négli- geables. Cette instrumentation pose la question suivante: comment concrètement apprend-t-on à utiliser cet outil? La prochaine section propose une réponse.

Un tiers des étudiants de formation initiale ou de formation continue du $3^{\mathrm{e}}$ cycle sont des porteurs de projet de création, soit 7 à 10 étudiants par an. Outre ce public mobilisé depuis 2003, une vingtaine de cas de créateurs a servi, au cours des années 2006 et 2007 , la construction et parfois l'expérimentation du protocole ici résumé. Ces cas sont, en partie, issus d'une thèse de doctorat en cours dont le cadre opératoire s'apparente à une recherche-action.

\section{Pertinence d'une approche par le BM dans l'accompagnement des porteurs de projet de création d'entreprise}

La tolérance à l'ambiguïté est l'une des principales difficultés face à laquelle les créateurs ne sont pas identiquement armés. Tant que faire se peut, la méthodologie doit premièrement s'appuyer sur des questions concrètes auxquelles, par ses réponses, le créateur a le sentiment de donner corps au concept de BM. Deuxièmement, elle aboutit à une visualisation qui possède un caractère émancipatoire, à l'instar de ce qu'ont pu constater par exemple les utilisateurs de la cartographie cognitive (voir Cossette et al., 2003). L'instrumentation comporte ainsi trois étapes.

La première liste un ensemble de questions à (se) poser. La figure 1 , de construction théorique, trouve ainsi une traduction pratique comportant d'une part le cheminement afférent à la constitution du BM (ressources, parties prenantes) et, d'autre part, celui de sa finalisation (conventions) ou, dans une certaine mesure, de son institutionnalisation. Le tableau 1 présente les 
Tableau 1 - Liste de questions pour aider la conception du BM

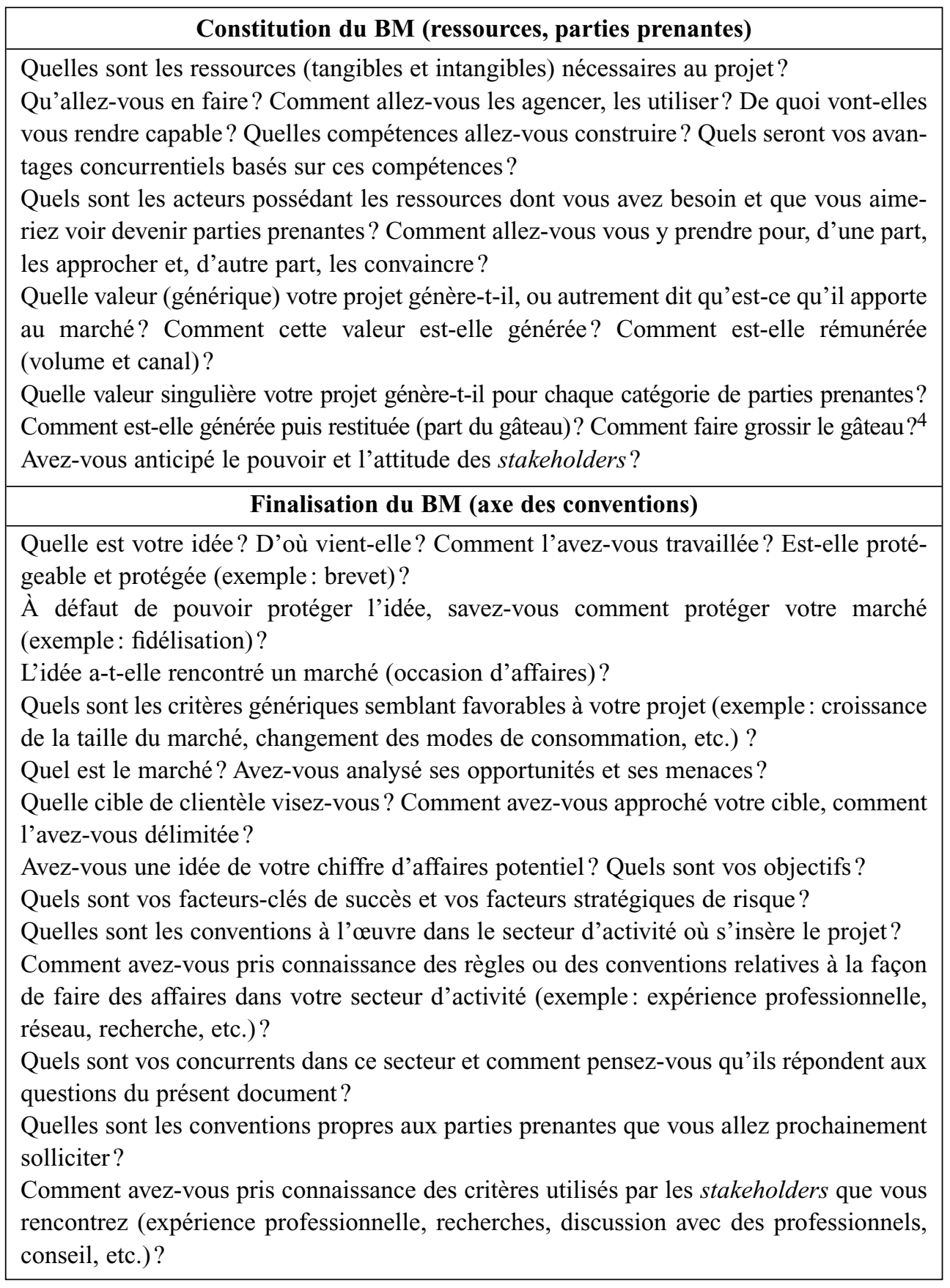

3. La métaphore du gâteau est utilisée pour montrer que l'ensemble des parties participe à la recette en apportant ses ressources et que chacun va récupérer une part du gâteau (ou plus exactement de la rémunération de celui-ci). 
questions posées par chaque axe du BM. On y retrouve certains éléments attendus dans un plan d'affaires, ce qui n'est pas surprenant puisque ce dernier doit, à notre sens, s'écrire autour du BM et l'ensemble des interrogations du tableau sert la construction de la convention d'affaires devant faire accord.

Chacune des questions fait elle-même l'objet d'une traduction en fonction du public concerné. Les étudiants suivant le troisième cycle, ainsi que certains porteurs de projet accompagnés dans le cadre de l'IRA (incubateur régional Aquitaine) ayant suivi les formations dispensées par l'équipe d'enseignants-chercheurs impliquée dans nos programmes, comprennent le vocabulaire utilisé. Par contre, les porteurs non formés ne subissent pas toujours les questions selon la formulation du tableau 1. Par exemple, la toute première question est formulée sans utilisation des mots « tangibles » et « intangibles », lesquels sont remplacés par des exemples (un savoir, de l'argent, un emplacement, des machines, un ingénieur, etc.). La question sur la valeur générique est reformulée pour être combinée à l'appréciation de l'opportunité ou du marché et les nuances apportées dans la formulation s'adaptent à la singularité du projet (et de son porteur).

La convention supposant un collectif, la deuxième étape de l'instrumentation consiste à remplir un tableau (voir un exemple avec le tableau 2). L'objectif de ce dernier est, partant des besoins du projet, d'aider l'identification des possesseurs de ressources, la compréhension de leurs attentes et de leur pouvoir, ainsi qu'à l'anticipation de leur attitude. Cette dernière renvoie aux conventions relatives à la catégorie de parties prenantes à laquelle ces pos- sesseurs appartiennent (exemple: les conventions dans le domaine du capital risque) mais aussi aux conventions du secteur investi (exemple: les ventes privées en ligne sur internet). Le créateur peut être conduit à procéder à un travail similaire à propos des concurrents. Si ceux-ci sont étudiés (voir les questions du tableau 1), et outre le travail de positionnement que la stratégie complètera, ils sont surtout vus comme des concurrents potentiels dans la captation de ressources dont le créateur a besoin. À ce titre, ce dernier est particulièrement sensibilisé au fait que la concurrence commence au niveau de l'obtention des ressources, chaque acteur du secteur (ou même d'autres secteurs) luttant pour obtenir les meilleures d'entre elles, qui ne sont ni inépuisables ni toujours disponibles. Ce type de tableau ne prétend pas poser toutes les questions relatives au réseau d'affaires mais incorpore l'essentiel pour construire la convention impliquant un réseau de représentations partagées d'une affaire. Le micro-ordinateur équipé d'un tableur est évidemment utile. Pour certains dossiers, un lien dynamique conduit, en un clic de souris, à une page spécifique de renseignements de la partie prenante concernée. Outre le travail du BM, l'un des grands avantage de ces outils est de permettre au porteur de projet de voir son réseau d'affaires, d'en prendre conscience, ce qui oblige, bien souvent, à entrer en contact avec les partenaires potentiels (la fréquence des contacts offrant à chaque partie une compréhension et une vérification de l'existence de la convention). Autrement dit, au confort de la réflexion menée face à un micro-ordinateur bien installé dans une pièce d'où on refait le monde, le candidat à l'entreprise doit substituer une rencontre 
réelle, sur le terrain, avec les acteurs concernés par son affaire. Certains créateurs ont un goût naturel pour l'action et les rencontres qu'occasionne celle-ci, mais il n'est pas rare que le contact soit vécu comme une contrainte comme c'est souvent le cas avec les étudiants. Ces derniers sont ainsi vite sensibilisés au besoin de se confronter à la réalité de leur projet.

Cette représentation en tableau est à la fois dynamique et modulable. Elle est dynamique car le tableau évolue en fonction du stade d'avancement du projet et de la rencontre avec les possesseurs de ressources, notamment lorsqu'ils deviennent des parties prenantes. Les étudiants se retrouvent alors face à une convention dynamique. La représentation est modulable, par exemple sous forme de carte comme le font sponta- nément certains porteurs de projet. Prochainement, une représentation par carte cognitive est envisagée, avec préalablement une recherche visant à apprécier l'utilité de cette forme de schématisation dans ce contexte.

Parmi la vingtaine de créateurs accompagnés, nous avons retenu comme illustration le cas de Monsieur H, créateur rencontré lors d'un séminaire de formation dispensé auprès des porteurs de projets bénéficiant des services de l'incubateur régional Aquitaine. Il a souhaité recevoir des conseils personnalisés. Les sept rendez-vous de travail, couvrant une période de quatre mois, chacun d'une durée d'environ trois heures, avaient comme objectif de conduire l'entrepreneur à concevoir progressivement son BM.

\section{CONTEXTE DU CAS FRUITS SÉCHÉS}

Le projet de Monsieur H s'inscrit dans la filière de production des fruits séchés, dont chaque acteur contribue à la qualité finale des produits destinés à la consommation, la mesure du taux d'humidité des fruits séchés étant un des critères d'évaluation de cette qualité. Pour faire cette mesure, il n'existe sur le marché qu'un procédé datant des années 1960 et utilisant un appareil importé des États-Unis qui n'a jamais donné pleine satisfaction. Une institution, nommée ici le « Bureau interprofessionnel du fruit séchés » (BIFS), s'est saisi de ce problème et, en tant que représentante de toute la profession, a décidé de trouver une solution à la nécessité d'une mesure fiable du taux d'humidité des fruits séchés.

Un spécialiste de la recherche de solutions innovantes, Monsieur H, a été mandaté par le BIFS pour travailler sur la résolution de ce problème. Monsieur $\mathrm{H}$ a pris contact avec un scientifique. Ce dernier, en collaboration avec un ingénieur évoluant au sein du même laboratoire universitaire, a imaginé une solution dont le dépôt de brevet est en cours (Monsieur $\mathrm{H}$ en sera temporairement le propriétaire, c'est-à-dire jusqu'à la création de la société, laquelle récupérera les droits). Le BIFS, séduit, encourage la poursuite du projet et participe, à la fois, au financement du prototype et au prétest de la solution en détachant 10 techniciens représentants de la filière. Un intérêt commun a conduit le BIFS et Monsieur H à retenir ce dernier comme porteur d'un projet de création d'entreprise de commercialisation de la solution technique de mesure du taux d'humidité des fruits séchés. L'idée travaillée, l'opportunité d'affaires a été évaluée, dont le potentiel dépasse le cadre de la seule demande du BIFS. 


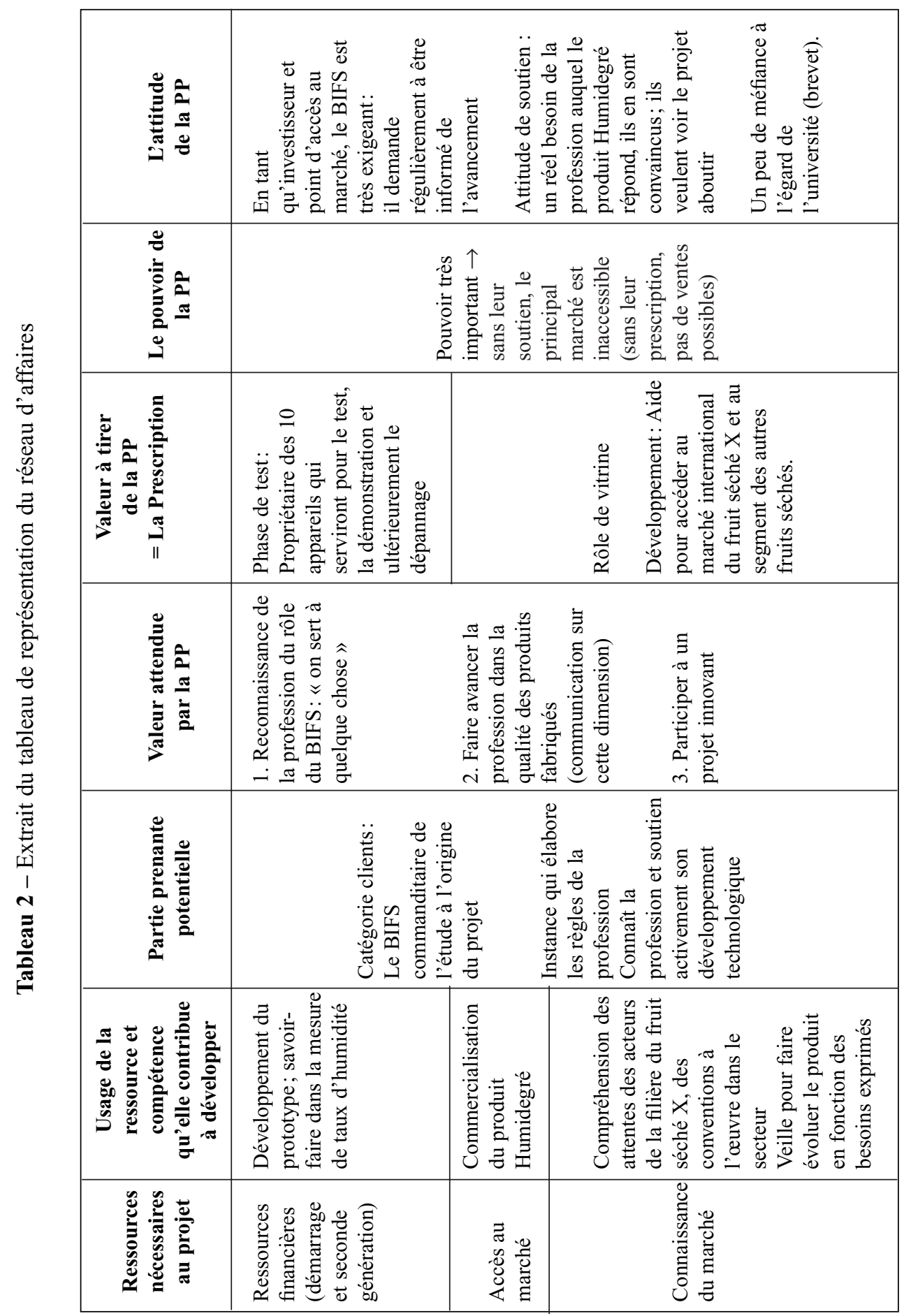


Nous reproduisons dans le tableau 2 (Extrait du tableau relatif au réseau), l'exemple de la partie prenante BIFS (nous ne livrons qu'un extrait du tableau car la reproduction totale, avec tous les possesseurs de ressources identifiés, prendrait quelques pages).

Derrière cette représentation synoptique permettant aussi de comprendre ce qui peut faire l'accord de tous, une vingtaine de tableaux singuliers aux parties prenantes a été remplie. Ces tableaux ont conduit le principal porteur, Monsieur $\mathrm{H}$, à comprendre d'autres registres conventionnels, à savoir ceux des parties prenantes ou, au moins, des catégories qu'ils intègrent (capital-risque, financeurs institutionnels, fournisseurs, etc. les typologies de parties prenantes proposées par la littérature pouvant être utilisées). Dans notre exemple, certaines conventions sont liées à la nature innovante du projet. Par exemple, il s'est avéré très utile pour Monsieur $\mathrm{H}$ de comprendre les exigences des institutions apportant des subventions en amont de la création effective de l'entreprise et de mesurer les conséquences, pour le projet, des relations qui existent entre ces différents acteurs de la valorisation de la recherche. Ainsi, outre les conventions du secteur, Monsieur H a compris qu'il convenait de composer avec les conventions des parties prenantes et que son BM ne pouvait convaincre sans intégrer les attentes et sans considérer à la fois le pouvoir et l'attitude des partenaires potentiels.

D'autres conventions sont inhérentes au marché de certains types de fruits séchés: les nombreuses rencontres et discussions avec le représentant du BIFS ont permis à Monsieur $\mathrm{H}$ d'assimiler les liens entre les acteurs de la filière et le rôle fédérateur de l'institution BIFS (on sait que la fréquence des contacts joue un rôle important dans la compréhension et la vérification de « l'existence » d'une convention).

Le remplissage des tableaux constitue une occasion de faire le point en synthétisant l'information essentielle. Dans une pratique d'accompagnement, la verbalisation est un exercice servant autant l'entrepreneur que l'accompagnateur, lequel ne peut prétendre connaître tous les secteurs d'activités et leurs acteurs. Idéalement, l'organisation d'une répétition orale avant certains rendezvous importants apporte au déploiement de l'exercice de conviction (ce que nous faisons avec nos créateurs).

Ce type de tableau permet également de sentir l'importance d'une ressource. Plus une ressource est stratégique (comme l'accès au marché dans le cas du BIFS), plus le pouvoir de la partie prenante est élevé, et plus son influence est grande sur le BM en cours de construction. Ainsi, c'est à la demande du BIFS que Monsieur $\mathrm{H}$ est devenu le principal porteur de projet ce qui a considérablement orienté le BM naissant. La collaboration aurait pu s'établir directement entre l'université et le BIFS mais cette décision a conduit à imaginer la création d'une entreprise commerciale pour exploiter l'innovation. Le BM n'est évidemment pas le même selon la nature du projet de valorisation $\mathrm{du}$ travail de recherche (exemple de différence de nature: dépôt de brevet par l'université et concession de licences à l'industrie, ou création d'entreprise sur la base du travail à valoriser). Monsieur H, en tant qu'une des principales parties prenantes, ne s'est pas exonéré de remplir un tableau pour lui-même, ainsi que pour ses deux associés (des scientifiques). Ce travail particulier visait à définir clairement les rôles, à identifier les 
attentes et les inquiétudes de chacun des membres de l'équipe. Ceci a constitué une étape dans la construction de la convention d'effort qu'est, en fait, l'entreprise naissante. En effet, les trois porteurs doivent fournir un effort commun pour, dans un premier temps, concevoir, produire et mettre sur le marché le produit Humidegré puis tous les autres produits de la société. Pour qu'ils puissent travailler efficacement, Monsieur H est conscient du besoin de partage d'une conception commune de la proposition de valeur et du BM constituant le cœur de ce que les stratèges appellent la vision stratégique (laquelle doit être un paradigme guidant les partenaires du projet). Il apparaît en effet nécessaire d'ali- gner le comportement des proches collaborateurs. Cela s'est avéré d'autant plus utile que, parmi les trois porteurs de projet, seul Monsieur $\mathrm{H}$ est issu du monde économique, les deux autres sont des scientifiques, étrangers aux réalités du monde des affaires. Monsieur H s'est servi des réflexions menées avec son conseiller, ici un chercheur, pour adapter son discours à ses collaborateurs afin de leur communiquer le plus efficacement possible le modèle d'affaires (la théorie de la traduction pourrait être convoquée).

Les parties prenantes ont également fait l'objet d'une représentation schématique (voir figure 3 , qui constitue la troisième étape de l'instrumentation).

Figure 3 - Schématisation simplifiée de la carte des parties prenantes

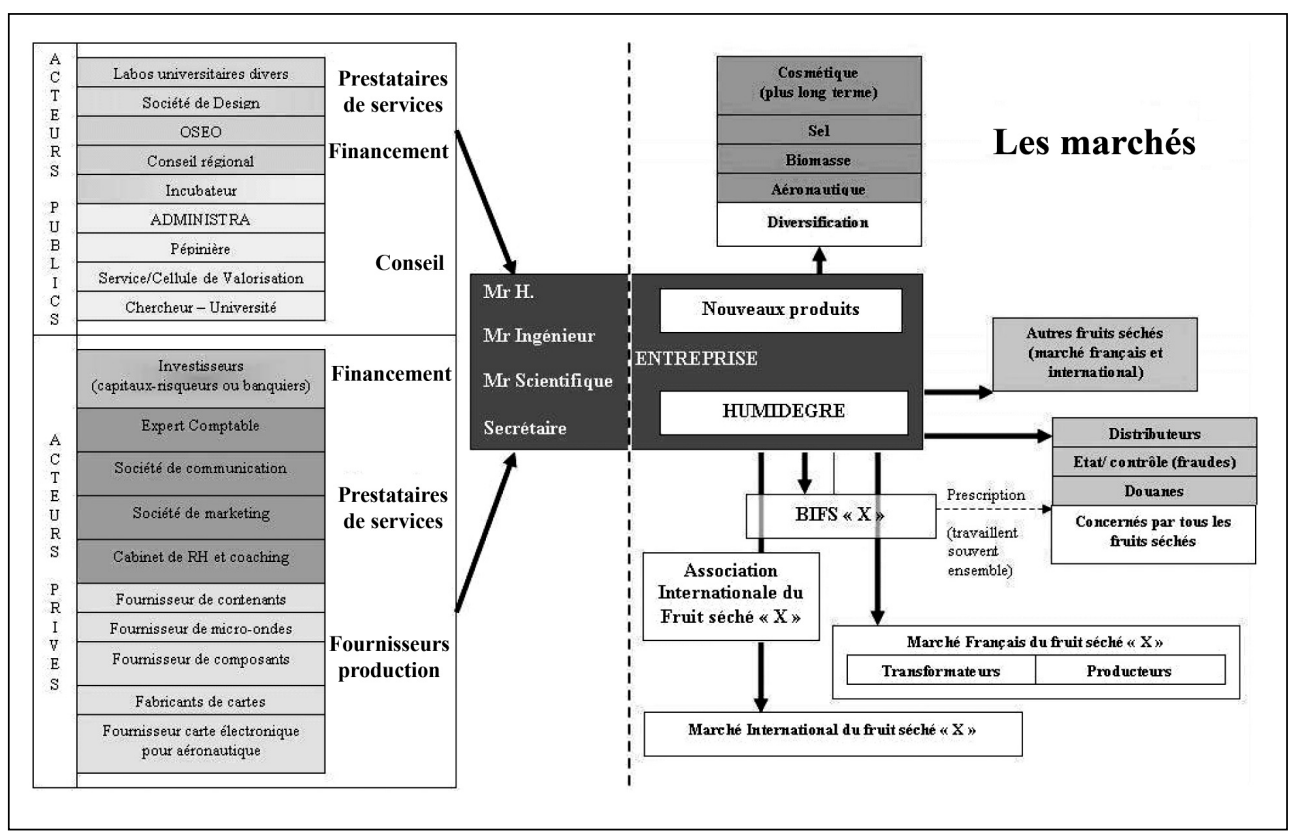


Sur la gauche de la figure 3, les apporteurs de ressources, devenus parties prenantes, contribuent à l'élaboration de la proposition de valeur de la future entreprise. Cette dernière repose sur une offre, à ce jour composée d'un produit unique, Humidegré, destiné au marché spécifique du fruit séché $\mathrm{X}$. Le BIFS ne figure pas dans cette liste car son rôle principal est celui de point d'entrée sur le marché de ce fruit séché. Il a donc été intégré à la partie concernant les différentes cibles de l'entreprise. S'agissant de cellesci, à court terme, le produit initial pourra être adapté à d'autres fruits séchés. Ultérieurement, le procédé scientifique à l'origine de la solution technique pour les fruits séchés fera l'objet de nouvelles applications et permettra à l'entreprise de toucher de nouveaux marchés très différents tels que l'aéronautique ou les cosmétiques. Le métier de l'entreprise reste, malgré cet élargissement de la cible, la mesure de taux d'humidité par procédé non destructif.

L'objectif de ce schéma n'est pas de dessiner totalement le BM de l'entreprise mais d'en fournir une représentation engageant les discussions. Plus synthétique que le tableau 2, il permet de visualiser le réseau d'affaires de la future organisation. L'ajout de couleurs et de différents types de flèches sont autant de détails visant à rendre cette représentation plus complète et plus riche en informations. Ainsi, les parties prenantes se répartissent selon qu'il s'agit d'acteurs privés ou institutionnels et se distinguent en fonction de la nature des ressources qu'elles apportent.

La distinction entre les deux catégories de parties prenantes (privées et institutionnelles) est fondée sur la nature différente des conventions de qualification partagées par les acteurs de ces deux sphères dis- tinctes. $\mathrm{Mr} \mathrm{H}$ a en effet souvent évoqué la difficulté pour lui de composer à la fois avec le milieu scientifique universitaire et des acteurs du monde économique.

Cette représentation, construite avec le porteur, est informatisée. À chaque partie prenante est associé un lien hypertexte donnant accès à des informations détaillées (contact, historique de la relation, pouvoir, etc.) complétant ce qui est fait avec le tableau 2.

\section{DISCUSSION - CONCLUSION}

Dans cette discussion-conclusion, nous revenons sur ce que le travail nous semble apporter en termes de lecture théorique du $\mathrm{BM}$ et de cadre pédagogique et pratique mis en place. Nous exposons ensuite ses limites tenant à sa focalisation sur le contexte de la création d'entreprise auquel le concept de BM nous semble fondamentalement attaché.

Ainsi, nous avons pu constater que sur le plan des ressources, le travail d'accompagnement fait apparaître la relative importance de chacune d'entre elles. En effet, si tout est à prendre en compte, les créateurs remarquent que chaque ressource est plus ou moins cruciale et que le pouvoir de leur possesseur est à nuancer. Sur le plan des parties prenantes, les expériences s'inscrivant dans le temps, leur influence est estimée de façon dynamique. Là encore une pondération des parties prenantes apparaît, souvent liée à la nature des ressources qu'elles détiennent ou à l'influence des conventions qu'elles partagent.

Sur le plan des conventions, centrales dans notre construction, les enseignements sont remarquables. Les discussions régulières avec un entrepreneur permettent d'observer comment il découvre et compose avec les 
conventions qui entourent le couple qu'il forme avec son projet. On peut également étudier comment il met en place le registre conventionnel autour duquel les détenteurs de ressources qu'il sollicite se rassemblent et s'entraînent. Pratiquement, les conseillers en création d'entreprise gagneraient eux-mêmes en compréhension des projets des créateurs accompagnés s'ils étaient formés au BM. Le temps consacré aux rendez-vous semble toutefois réserver le financement de ce type de conseil aux projets à fort potentiel et pour lesquels le $\mathrm{BM}$ comporte de nombreuses dimensions à considérer mais sans perdre de vue l'essentiel du projet. Sa mise au point apporte à la fois une maîtrise et une assurance de ce qui concerne le cœur de l'affaire. On remarque que la finalisation du BM diminue l'influence des parties prenantes ensuite rencontrées sur les caractéristiques du projet. En effet, à un certain stade de mise au point du BM, après avoir apprécié les attentes et les pouvoirs des parties prenantes (y compris leurs conseillers), les créateur arrêtent en quelque sorte leur BM et ne l'amendent plus, ou très timidement. Pour utiliser une métaphore culinaire, la recette du gâteau est arrêtée, les possesseurs de ressources sontils alors prêts à franchir le pas, à aider le gâteau à grossir pour mieux le partager? En fait, la convention semble se stabiliser, ce qui ne stoppe pas pour autant son évolution. Par exemple, le lancement effectif de l'entreprise et la confrontation avec la réalité des affaires constituent un autre parcours influençant le BM. Si ce dernier est apparu en contexte entrepreneurial, il devient stratégique pour l'entreprise établie et remet au goût du jour les travaux sur les relations stratégie-structure, puisque la configuration organisationnelle (dont les frontières dépassent celles de l'entité naissant du phénomène entrepreneurial) matérialise, en quelque sorte, le BM.

Outre notre perception, les apports du travail s'apprécient également par les retours formulés par les protagonistes du projet pédagogique et ceux de la mise au point des projets concrets.

S'agissant de la pédagogie, une première réaction a été mesurée par le jury de soutenance des plans d'affaires déposés par les étudiants du $3^{\mathrm{e}}$ cycle. Ce jury est, à peu de chose près, composé depuis plusieurs années des mêmes personnes. Il a été remarquable de constater que, sans être avertis du changement de pédagogie et de l'importance nouvellement accordée à l'élaboration d'un BM, à l'unanimité les membres ont évalué très positivement et spontanément la clarté avec laquelle les étudiants présentaient leur projet ${ }^{5}$. Ce sentiment s'est confirmé l'année suivante.

Une deuxième réaction concerne l'équipe pédagogique, notamment celle du $3^{\mathrm{e}}$ cycle qui s'est explicitement prononcée sur ce sujet. Les enseignants appréhendent mieux leur implication et l'impact de leur prestation dans le projet pédagogique par l'ancrage de celui-ci dans un processus offrant du liant dans ce qui était auparavant davantage une juxtaposition d'interventions. Une autre manifestation de la satisfaction des

5. Les étudiants soutiennent leur plan d'affaires devant deux ou trois membres. Sur la même demi-journée, plusieurs jurys opèrent en parallèle. La douzaine de membres du jury est réunie après la soutenance pour un compte rendu. 
enseignants s'est révélée par une disponibilité accrue auprès des étudiants, par exemple par des prises de rendez-vous dans la mise au point de leur projet. Il n'est peutêtre pas déraisonnable de considérer que le $\mathrm{BM}$ joue déjà son rôle de cristallisation entre parties prenantes, ici les étudiants et leurs enseignants (des possesseurs de ressources cognitives devenus des parties prenantes conseillers).

Un troisième ensemble de réactions est bien évidemment celui des étudiants qui, indubitablement, ont gagné en compréhension dans le montage d'une affaire, notamment sur ce qui constitue son cœur tout en comprenant qu'on ne vend pas une affaire comme on vend un produit. Leur créativité est canalisée par les réactions du terrain et devient plus raisonnée et moins naïve. Les formations dispensées font l'objet d'une évaluation à l'aide de grilles remplies anonymement par les étudiants. Les résultats sont très positifs. Un constat identique émerge des porteurs de projet de création hébergés par l'incubateur régional pour lesquels un programme spécifique est dispensé.

Les étudiants de troisième cycle mesurent concrètement l'aide à l'élaboration et à l'expression du BM. La première version (puisqu'il est appelé à évoluer) étant validée, l'étudiant étant rassuré et prenant confiance, l'apprentissage peut se reporter vers les autres éléments de mise au point de l'affaire, notamment la vision stratégique (avec des pôles de travail concernant le montage juridico-financier, les systèmes de gestion, etc.) et l'écriture de celle-ci dans un plan d'affaires. Les anglo-saxons parlent du business plan comme d'un « selling document », mais ce qui est à vendre est à notre sens le BM.
S'agissant de l'accompagnement pratique des porteurs de projet, un premier constat satisfaisant est la compréhension des créateurs à l'égard de l'intérêt à travailler le $\mathrm{BM}$, dont il faut les aider à saisir le sens, ce qui ne pose finalement pas de problème fondamental. Le propos est certes adapté (ou traduit...) et le BM devient un objet avec lequel ils sont à l'aise. Cela provient sans doute de son fort ancrage dans le problème concret du rassemblement des ressources auquel sont confrontés les entrepreneurs.

Certains porteurs ayant participé à la phase empirique de la recherche sont accompagnés par ailleurs par des organismes spécialisés. L'accompagnement repose sur l'aide au montage du plan d'affaires, laquelle comprend essentiellement l'évaluation de l'adéquation porteur-projet, le soutien dans la réalisation de l'étude de marché et dans la réalisation du prévisionnel financier. Le travail sur le BM, ancré dans le problème du rassemblement des ressources, ne semble pas suffisamment inscrit dans les programmes d'accompagnement et de conseil, ou est alors réduit à la levée de fonds. Avec le BM, les échanges de valeurs entre parties prenantes sont compris et mieux négociés. Les tableaux servent cette compréhension. Mais au-delà de l'identification des parties prenantes et des ressources, l'outillage mis en œuvre permet aux entrepreneurs d'appréhender les conventions de qualification partagées par les parties prenantes dont ils doivent obtenir l'adhésion, par exemple les conventions du secteur d'activité mais également celles du milieu de la création d'entreprise.

Enfin, la réflexion en termes de BM conduit à préciser le modèle de revenus à l'origine de la valeur générique et à verbaliser 
l'échange de valeur spécifique entre la future organisation et chacune des parties prenantes. Ce travail sert les exercices de conviction déployés (certains créateurs ont par exemple intégré à leur plan d'affaires des tableaux et schémas issus du travail avec le chercheur). Il leur permet également, comme dans le cas de $\mathrm{Mr} \mathrm{H}$, de faciliter l'alignement des proches collaborateurs autour d'une vision partagée de la proposition de valeur, étape nécessaire à la naissance de la convention d'effort.

Une des limites tient au contexte de la création d'entreprise auquel se circonscrit notre travail. Il est sans doute utile de le dépasser pour poser la question de l'utilité du BM pour la stratégie de l'entreprise établie, comme le font Lecocq et al. (2006). Selon ces auteurs, le BM se place à un niveau meso conduisant à la mise en œuvre de nouvelles formes d'organisation de la chaîne et du réseau de valeur. Ceci est particulièrement valable dans le cadre d'un entrepreneuriat persistant où les entreprises créées doivent être managées. Les chercheurs en entrepreneuriat et en stratégie sont appelés à se côtoyer pour produire sur l'entrepreneur stratège et les outils utiles à celui-ci, en y associant les spécialistes du marketing pour la conceptualisation de l'offre.

L'autre limite majeure concerne l'exercice de conviction, lequel appelle la mise en place d'un protocole visant à mesurer l'impact effectif du travail de construction du BM sur la capacité à convaincre. Les outils aident l'entrepreneur à " penser » son modèle d'affaires, la présence du chercheur lui donnant aussi l'occasion de verbaliser celui-ci et lui permettant de se poser des questions qu'il n'aurait pas abordé seul. Au-delà de l'apport en termes de structuration du projet de création, le travail sur le BM est un soutien pour l'entrepreneur dans le cadre des exercices de conviction qu'il est amené à déployer. En effet, la prise en considération systématique de l'échange de valeur lui permet de savoir quels aspects de son projet mettre en avant lorsqu'il doit remporter l'adhésion du détenteur de ressources rencontré. Mais il est nécessaire de mettre en place une recherche pour véritablement pouvoir affirmer que l'exercice est « meilleur » avec notre outillage que sans. Le travail est en cours, mobilisant les cadres théoriques afférents.

\section{BiBLIOGRAPHIE}

Birley S., Westhead P., "A comparison of new firms in assisted and non assisted aeras in Great Britain", Entrepreneurship and Regional Development, vol. 4, n 4, 1992, p. 299-338.

Cossette P., Audet M., «Qu'est-ce qu'une carte cognitive? », Cartes cognitives et organisations, Cossette P. (dir), Les Éditions de l'ADREG, 2003 (http://www.adreg.net)

Desreumaux A. (dir.), Les stratégie des entreprises en tant qu'acteurs de l'emploi, CLAREE - IFRESI, Rapport au ministère du Travail et de l'Emploi et de la Formation professionnelle (DARES), janvier 1995. 
Dupuy J.P., Eymard-Duvernay F., Favereau O., Orléan A., Salais R., Thévenot L., « Introduction du dossier sur les conventions », Revue Économique, vol. 40, n 2, 1989.

Gomez P. Y., Qualité et théorie des conventions, Economica, Paris, 1994.

Gomez P.Y., Le gouvernement de l'entreprise, InterEditions, Paris 1996.

Gomez P.-Y.« Information et conventions : le cadre du modèle général », Revue française de gestion, $\mathrm{n}^{\circ} 112$, janvier-février 1997, p. 217-239.

Gomez P.Y., Jones B.C, “Convention: an interpretation of deep structure in organization", Organization Science, vol.11, n 6, 2000, p. 696-708.

Jouison E., « Délimitation théorique du Business Model », XIV Conférence Internationale de Management Stratégique (AIMS) à Angers, Juin 2005.

Lecocq X., Demil B., Warnier V., « Le business model, un outil d'analyse stratégique », L'Expansion Management Review, ${ }^{\circ} 123,2006$, p. 96-109.

Munier B., Orléan A., « Sciences cognitives \& sciences économiques et de gestion », GRID CREA, 1993.

Orléan A., Analyse économique des conventions, Presses Universitaires de France, Paris, 1994.

Ughetto P. «L'entreprise dans l'économie des conventions », Revue Économique, vol. 51, $\mathrm{n}^{\circ} 1$, janvier 2000, p. 51-73.

Verstraete T., Entrepreneuriat - connaître l'entrepreneur, comprendre ses actes, L'Harmattan, 1999.

Verstraete T., Proposition d'un cadre théorique pour la recherche en entrepreneuriat, Editions de l'ADREG (http://www.adreg.net), 2003.

Verstraete T., Saporta B., Création d'Entreprise et Entrepreneuriat, Éditions de l'ADREG (http://www.adreg.net), 2006. 